\title{
X-ray Phase Imaging by Two-Beam Interferometry with Full-Field X-ray Microscope and Transmission Grating
}

\author{
$\underline{\text { Atsushi Momose }}^{1,2, *}$, Hidekazu Takano ${ }^{1}$, Yanlin $\mathrm{Wu}^{1}$, Karol Vegso ${ }^{2}$, Yasuko Terada ${ }^{2}$, and Masato \\ Hoshino $^{2}$ \\ 1. Institute of Multidisciplinary Research for Advanced Materials, Tohoku Univ., Sendai, Japan. \\ 2. JASRI/SPring-8, Sayo, Japan. \\ atsushi.momose.c2@tohoku.ac.jp
}

X-ray phase imaging has been developed extensively by various techniques for generating X-ray phase contrast. An important aspect of X-ray phase imaging is that the information on X-ray attenuation, X-ray phase shift (or refraction), and more recently X-ray scattering is obtained quantitatively by processing digitally acquired phase-contrast image(s). Therefore, X-ray phase imaging is highly compatible with Xray computed tomography for three-dimensional observations. Among the methods of X-ray phase imaging, a Bonse-Hart crystal interferometer was used for the first successful X-ray phase imaging/tomography [1]. However, the spatial resolution of BHI-based X-ray phase imaging is limited around $10 \mu \mathrm{m}$. Nevertheless, BHI is unique in that the low-spatial-frequency (even zero-frequency) Xray phase shift is detectable, while other methods are only sensitive to refraction (differential phase). Therefore, we have studied to explore a better spatial resolution by building a two-beam interferometer in a full-field X-ray microscopy configuration.

The optical system constructed in this study is similar to that developed by combining grating-based Xray phase imaging and X-ray microscopy (Fig. 1). The Talbot interferometer shown in Fig. 1(a) produces magnified differential-phase images [2]. When the inverse geometry is selected, the second grating (G2) of the Talbot interferometer can be omitted (Fig. 1(b)), and a twin phase image is generated, which is a superposition of two phase image of opposite sign with a finite shear distance [3]. While the shear distance is smaller than the system spatial resolution in differential phase images, the relatively large distance between the phase grating (G1) and the image plane makes the shear distance much larger than the resolution. It is understood that two-beam interferometry is established locally. So, if the shear distance is enlarged, high-resolution direct phase imaging must be feasible, as illustrated in Fig. 1(c). Assuming that a plane wave illuminates a FZP, a half of the wave is used as an object beam and the other half is for a reference beam. A phase grating is placed near the downstream of the focal plane. Two-beam interference occurs between the $0^{\text {th }}$ order diffraction of the object beam by the grating and the $1^{\text {st }}$ order diffraction of the reference beam. The interference between the $-1^{\text {st }}$ order of the object beam and the $0^{\text {th }}$ order of the reference beam produces another phase image of opposite sign at separated position. Note that the phase image is generated through the fringe scan (phase stepping) procedure; that is, interference images are acquired by displacing the grating at a sub-period step. Since the displacement does not affect the $0^{\text {th }}$ order, the phase difference between the object and reference beams is given robustly, producing $2 \pi$ phase difference by one-period grating displacement.

The shear distance is inversely proportional to the grating period, and therefore the fabrication of a small-period grating is a key issue for this idea. When the grating period is twice of the outermost zone width of the FZP, the shear distance is the same as the diameter of the FZP. In this study, however, we preliminarily used a phase grating of a period of $2.38 \mu \mathrm{m}$ for demonstration. The experiment was performed at BL37XU, SPring-8 at $9 \mathrm{keV}$. We used a FZP whose diameter was $750 \mu \mathrm{m}$ and outermost 
zone width was $200 \mathrm{~nm}$, and a full-field microscope with a magnification of 23.3 was build. A ten-step fringe scan was performed with a 1-s exposure each. Figure 2 shows phase images of test chart and a plastic fiber with polystyrene spheres $(3 \mu \mathrm{m}$ in diameter). The shear distance achieved in this measurement was $125 \mu \mathrm{m}$, and the phase images expected by this idea are indicated by arrows in Fig. 2. Phase unwrapping has not been carried out. The artifact image appeared in the middle of them, which should be cancelled out, is a problem unsolved. We speculate that asymmetric diffraction (including higher-order diffraction) by the phase grating caused this feature. The evaluation of the phase grating should be performed separately.

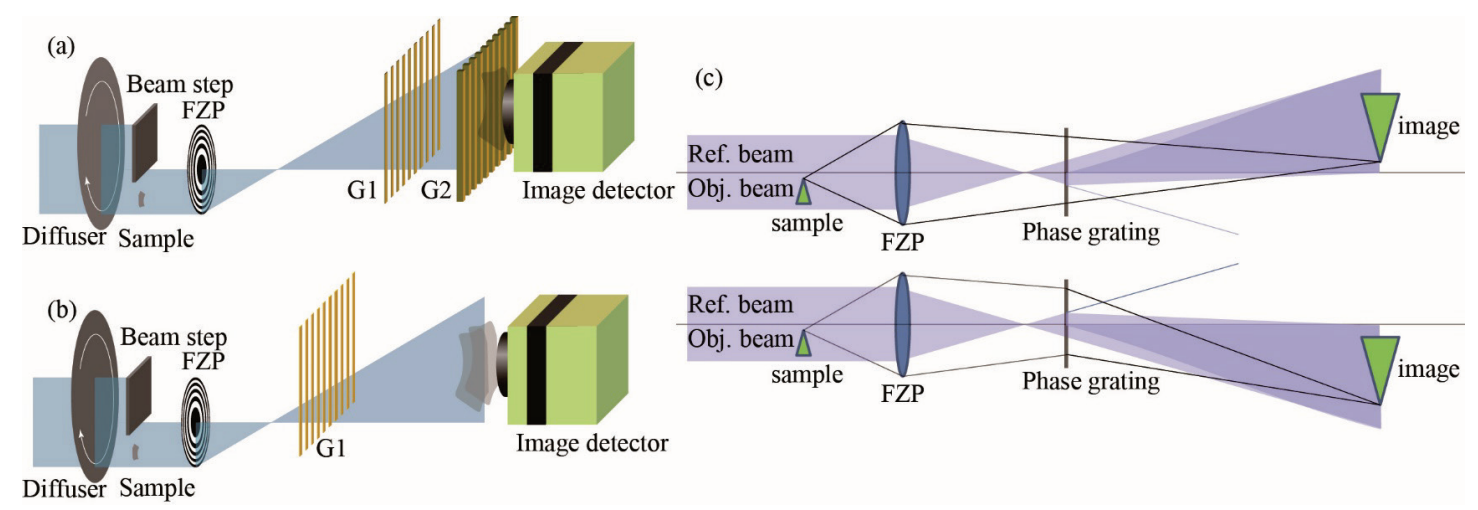

Figure. 1. Combination of a full-field X-ray microscope and (a) Talbot interferometer (G1 and G2) [2], (b) single grating (G1) [3], and (c) grating-based two-beam interferometer.

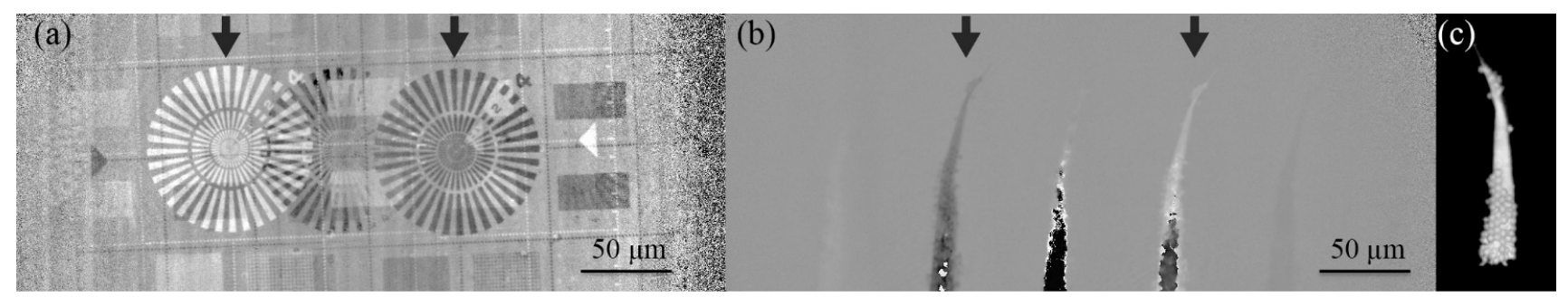

Figure. 2. Result of direct phase imaging of (a) test chart and (b) plastic fiber is polystyrene spheres. The arrows indicate the expected phase images, while the problem of artifact images is unsolved. (c) Rendering view of the phase tomogram reconstructed for the sample (b).

Apart from this problem, the phase image of the sample is isolated from the artifact, and we tested phase tomography by acquiring phase images at 450 angular positions over 180 degrees, as shown in Fig. 2(c). Resultant spatial resolution was $500 \mu \mathrm{m}$. Although there was a problem of the remaining artifact, this approach is promising for robust X-ray phase imaging by two-beam interferometry in combination with FZP optics in comparison with some earlier trials [4]. We expect that this approach is feasible for nanoscopic phase tomography with a narrow-pitch phase grating.

This study was supported by JST ERATO (JPMJER1403). The experiment was performed under the approval of the SPring-8 committee (2017A1360).

[1] A Momose, NIMA 352 (1995) 622-628

[2] Y Takeda et al, Appl. Physics. Express 1 (2008) 117002

[3] W Yashiro et al, Phys. Rev. Lett. 103 (2009) 180801

[4] A Momose, Microscopy 66 (2017) 155 